\title{
Clinical Effect of Electroacupuncture Baihui and Neiguan Acupoint on Cognitive Function Recovery in Elderly Patients after General Anesthesia
}

\author{
Yuming Zhang', Haiyan Huo ${ }^{2}$, Zhu Zhu' ${ }^{2}$, Qifan Huo ${ }^{1}$, Jing Zhao ${ }^{1}$, Jun Wang ${ }^{3 *}$ \\ ${ }^{1}$ Department of Anesthesiology, Shaanxi Provincial People's Hospital, Xi'an 710068, Shaanxi Province, China \\ ${ }^{2}$ Operation Room, Shaanxi Provincial People’s Hospital, Xi’an 710068, Shaanxi Province, China \\ ${ }^{3}$ Department of Anesthesiology, Shaanxi Provincial Cancer Hospital, Xi'an 710061, Shaanxi Province, China \\ Funding: Natural Science Basic Research Program of Shaanxi Province ( No.: 2020JQ-950 ).
}

\begin{abstract}
[Abstract] Objective: To study and analyze the clinical effect of electroacupuncture at Baihui and Neiguan Points on cognitive function recovery of elderly patients after general anesthesia. Methods: The subjects were 80 elderly patients who received general anesthesia from January 2019 to February 2021. According to different anesthesia methods, they were divided into research group (electroacupuncture at Baihui and Neiguan combined with conventional drug anesthesia) and control group (simple drug anesthesia). The postoperative cognitive function recovery of the two groups was observed and compared. Results: Compared with the control group, the MMSE score and POCD incidence of research group were significantly better. There was significant difference between the two groups $(\mathrm{P}<0.05)$. Conclusion: Conclusion electroacupuncture at Baihui and Neiguan combined with conventional drug anesthesia can promote the recovery of cognitive function and prevent cognitive dysfunction in elderly patients under general anesthesia.
\end{abstract}

Key words: Electroacupuncture; Baihui; Neiguan point; Elderly patients; General anesthesia; Postoperative cognitive dysfunction; Clinical effect

Publication date: May, 2021; Publication online: 31 May, 2021

*Corresponding author: Jun Wang, zhangyum2021@163.com

\section{Introduction}

Elderly patients are relatively special. When receiving general anesthesia, because of their poor drug tolerance and low sensitivity, the incidence of postoperative complications is high. Among all kinds of complications, the most common one is cognitive dysfunction. When cognitive impairment occurs, the patient is manifesting mental disorder. Memory impairment. And through TCM manipulation to stimulate related acupoints can effectively prevent or improve the problem of cognitive impairment ${ }^{[1]}$. The research content of this paper is the clinical effect of electroacupuncture at Baihui and Neiguan Points on cognitive function recovery of elderly patients after general anesthesia.The results were as follows.

\section{Data and methods}

\subsection{General information}

In this study, 80 elderly patients who received general anesthesia in our hospital were selected and randomly divided into two groups. In 40 cases of control group, the ratio of male to female was $4: 1$. The average age was $(65.9 \pm$ 6.4) years. The ratio of male to female of 40 patients in research group was 5:1. The average age was $(66.4 \pm 8.1)$ years. There was no significant difference in age and gender between the two groups ( $\mathrm{P}>00.05)$. This study was officially carried out with the permission of the hospital ethics committee. 


\subsection{Inclusion and exclusion criteria}

Selected patients have been informed of the study and will voluntarily cooperate to complete all studies and sign informed consent; The patient has no other serious tissue or organ disease and has normal consciousness; Excluding other serious tissue or organ diseases, refusing to participate in the study, and having difficulty in clearly and autonomously answering the research-related questions raised by the researcher, the patient's family strongly rejected the study, could not answer the questions specifically, and withdrew at the middle of the study (e.g. metastasis or death).

\subsection{Anesthesia methods}

Half an hour before anesthesia induction, the patients of research group were selected to point Baihui and Neiguan for transcutaneous electrical stimulation and maintained until the end of the operation. The electrical stimulation was connected to the acupuncture anesthesia instrument, the dense wave was selected, and the frequency was adjusted to $2 / 100 \mathrm{~Hz}$, which was appropriate to the patient's tolerable intensity. All patients were intubated with etomidate $0.2 \mathrm{mg} / \mathrm{kg}$, sufentanil $0.2 \mathrm{ug} / \mathrm{kg}$ and cisatracurium $0.2 \mathrm{mg} / \mathrm{kg}$. Sevoflurane inhalation combined with remifentanil was used to maintain anesthesia during the operation. Muscle relaxants were added intermittently during the operation to ensure the blood pressure and heart rate of patients in a stable state. At the end of the operation, the tracheal tube was pulled out after the patient was conscious in PACU.

\subsection{Observation items and indicators}

(1) All patients received Mini Mental State Examination (MMSE) score 1 day before anesthesia and 2, 4, 6 days after operation to evaluate the orientation, attention and calculation ability of patients. The full score is 30 . The higher the score, the lighter the cognitive impairment. (2) The incidence of POCD in the two groups was statistically analyzed on the 2nd, 4th and 6th day after operation.

\subsection{Statistical methods}

The data and information involved in this study were analyzed and processed by SPSS 20.0 statistical software.

\section{Results}

\subsection{MMSE scores were compared between the two groups at different time.}

There was no significant difference in the scores of the two groups before operation $(\mathrm{P}>0.05)$, and there was no statistical significance. The scores of the two groups increased from a lower level after operation, and the research group scores were significantly higher than those of the control group, and the effective difference between the two groups was statistically significant $(\mathrm{P}<0.05)$. See Table 1 for details.

Table 1. Comparison of MMSE scores of the two groups at different times ( points, $\overline{\mathrm{x}} \pm \mathrm{s}$ )

\begin{tabular}{|c|c|c|c|c|}
\hline Group & $\begin{array}{c}\text { One day before } \\
\text { operation } \\
\end{array}$ & 2 days after operation & 4 days after operation & $\begin{array}{c}6 \text { days after } \\
\text { operation }\end{array}$ \\
\hline $\begin{array}{l}\text { Research group } \\
\qquad(\mathrm{n}=40)\end{array}$ & $27.48 \pm 1.14$ & $24.38 \pm 1.56$ & $26.52 \pm 2.13$ & $27.38 \pm 3.11$ \\
\hline $\begin{array}{l}\text { Control group } \\
\qquad(\mathrm{n}=40)\end{array}$ & $27.45 \pm 1.16$ & $18.86 \pm 2.62$ & $22.57 \pm 2.11$ & $26.06 \pm 1.12$ \\
\hline
\end{tabular}

3.2 Comparison of the incidence of POCD in different postoperative time between the two groups The statistical results showed that the incidence of POCD in the research group was significantly lower than that in the control group at different time after operation, and the difference was statistically significant $(\mathrm{P}<0.05)$. See Table 2 for details.

Table 2. Comparison of POCD incidence in two groups at different time after operation (n, \%)

\begin{tabular}{cccc}
\hline Group & 2 days after operation & 4 days after operation & 6 days after operation \\
\hline $\begin{array}{c}\text { Research group } \\
(\mathrm{n}=40)\end{array}$ & $16.25 \%(13 / 40)$ & $6.25 \%(5 / 40)$ & $0.0 \%(0 / 40)$ \\
\hline
\end{tabular}




\begin{tabular}{|c|c|c|c|}
\hline $\begin{array}{l}\text { Control group } \\
\qquad(\mathrm{n}=40)\end{array}$ & $31.25 \%(25 / 40)$ & $15.0 \%(12 / 4)$ & $5.0 \%(4 / 40)$ \\
\hline
\end{tabular}

\section{Discussion}

POCD refers to the central nervous system complications in the elderly after surgery, which are manifested as mental disorder, anxiety, personality changes and memory impairment, which will affect the surgical treatment effect, increase the postoperative mortality, prolong the recovery time, increase the cost of diagnosis and treatment, and even have adverse effects on the quality of life of patients after hospitalization. Elderly patients are particularly prone to $\mathrm{POCD}^{[2]}$. At present, the pathogenesis of POCD has not been fully studied in clinic. Now the more unified view is that POCD has the same pathogenesis as Alzheimer's disease. On the basis of nervous system aging, and external factors such as anesthesia and surgery aggravate the degree of aging, which leads to the onset of POCD under the combined action of many factors, because all kinds of drugs before and during anesthesia can cause the onset of POCD It may lead to the changes of dopamine and acetylcholine in the nervous system, so it may also lead to POCD ${ }^{[3-4]}$.

In clinical research, POCD of elderly patients is often carried out through MMSE. When the MMSE score of patients after operation is reduced by more than 2 points compared with that before operation, it indicates that the cognitive function of patients is reduced. The results of this study show that the MMSE score of patients is reduced within one week after operation, which indicates that anesthesia and manual intervention may have adverse effects on the cognitive ability of patients. In order to avoid or reduce the incidence of POCD in elderly patients, we need to pay attention to brain protection in addition to perioperative nursing and improving anesthesia technology ${ }^{[5]}$. In the field of traditional Chinese medicine, acupoint stimulation has a significant effect on analgesia and control of physiological disorders, maintaining the physiological balance of patients and improving comfort. In the traditional acupuncture theory, our medical staff try to carry out acupuncture anesthesia, the effect is very significant, which shows that acupuncture can effectively regulate the internal environment of the human body, so that the body has a higher level of defense ability, and the anesthesia effect is relatively significantly improved ${ }^{[6]}$. Through a large number of clinical studies, it is found that acupuncture at Baihui and Neiguan Points has a significant effect on improving brain hypoperfusion, enhancing brain metabolic function, enhancing brain blood flow and restoring brain nerve cell function. In addition, acupuncture at Zusanli and Hegu can also be used during practical application, in which acupuncture at Zusanli can activate most brain regions, including frontal lobe, temporal lobe, insular lobe and part of cerebellum ${ }^{[7]}$. Among them, the most significant effect is to stimulate the temporal lobe region of the brain. By stimulating this region to regulate the body movement, sensation and language, acupuncture at Hegu has significant analgesic and sedative effects ${ }^{[8]}$.

The results of this study showed that the MMSE scores of research group patients receiving electroacupuncture at Baihui and Neiguan Points Combined with conventional drug anesthesia at different time after operation were significantly higher than those of control group patients receiving simple drug anesthesia $(\mathrm{P}<0.05)$ The incidence of group POCD was significantly lower than that of the control group ( $\mathrm{P}<0.05)$, which confirmed that electroacupuncture at Baihui and Neiguan combined with conventional drug anesthesia can achieve the expected comprehensive effect in elderly patients under general anesthesia. However, it should be noted that because the number of included samples in this study is small, the conclusion may not be absolutely convincing. It is expected that large sample studies can be carried out in the future, which can increase the persuasiveness of the research conclusions and reduce the errors.

In summary: Conclusion electroacupuncture at Baihui and Neiguan combined with conventional drug anesthesia can promote the recovery of cognitive function and prevent cognitive dysfunction in elderly patients under general anesthesia.

\section{References}

[1] Wu JH, Shi J, Chen YX, et al. Effect of intraoperative electroacupuncture on postoperative delirium in elderly patients [J]. Journal of Tianjin Medical University, 2020,26 (5): 466-470

[2] Zhang CL, Zhu LL, Yan D. Effect of Electroacupuncture on cognitive function recovery of elderly patients after general anesthesia [J]. Shanghai 
Journal of Acupuncture and Moxibustion, 2015(2): 132-133.

[3] Wang JB. Study on the effect of electroacupuncture therapy of traditional Chinese medicine on the recovery of cognitive function in elderly patients after general anesthesia [J]. Chinese Modern Distance Education of traditional Chinese Medicine, 2017, 15 (7): 117-118.

[4] Zhu LL. Clinical study on the effect of acupuncture on cognitive function recovery of elderly patients after general anesthesia [D]. Shanghai: Shanghai University of Traditional Chinese Medicine, 2013.

[5] Yin ZL, Meng ZX, Lin SY, et al. Effect and mechanism of acupuncture on postoperative cognitive dysfunction in elderly rats $[\mathrm{J}]$. Journal of Acupuncture Clinical, 2015, (12): 68-70.

[6] Tang Y, Wang T, Yang L, et al. Acupuncture for post-operative cognitive dysfunction: a systematic review and meta-analysis of randomized controlled trials[J]. Acupuncture in Medicine. 2020.

[7] Qiao L, Guo M, Qian J, et al. Research advances on acupuncture analgesia[J]. Am J Chin Med. 2020;48(2):245-258.

[8] Patil S, Sen S, Bral M, et al. The Role of acupuncture in pain management[J]. Curr Pain Headache Rep, 2016, 20(4): 22 . 